\title{
The Scalpel in the Management of Drug-Resistant Tuberculosis: Underrated and Underutilized
}

\author{
Verma $\mathbf{P}^{1}$, Dewan $\mathbf{R K}^{2}$, Purwar $\mathbf{P}^{3}$ and Sharma $\mathrm{A}^{4 *}$ \\ ${ }^{1}$ Senior Resident, TB \& RD, National Institute of Tuberculosis and Respiratory Diseases \\ (NITRD), India \\ ${ }^{2}$ Director and Senior Thoracic Surgeon, National Institute of Tuberculosis and Respiratory \\ Diseases (NITRD), India \\ ${ }^{3}$ Thoracic Surgeon, National Institute of Tuberculosis and Respiratory Diseases (NITRD), India \\ ${ }^{4}$ Chest Specialist, National Institute of Tuberculosis and Respiratory Diseases (NITRD), India
}

Short Communication

Volume 6 Issue 1

Received Date: April 16, 2021

Published Date: April 28, 2021

DOI: 10.23880 /oajprs-16000136

*Corresponding author: Amit Sharma, Senior Chest Physician, National Institute of Tuberculosis and Respiratory Diseases (NITRD), India, Tel: 9871935758; Email: dramitsharma55@yahoo.co.in

\section{Short Communication}

The human race has rarely encountered such a pestilent and persistent adversary as Tuberculosis (TB). From ancient times, this disease is inflicting its damage to mankind by causing morbidity as well as death to the afflicted. With time its management and control have both undergone extreme changes.

Initially when drugs had not been discovered, ample bed rest, good diet and a healthy environment were the only tools which were exercised with the hope of Spontaneous Cure. Surgey also played some role with early surgical therapies consisting of a variety of collapse therapies including thoracoplasty, ball plombage, and induced pneumothorax [1].

However, beginning with the discovery of Injection Streptomicin, a momentous revolution in the management of this terrible malady took place. With the advent of drugs such as Isoniazid, Rifampicin, Pyrazinamide etc. there was a great improvement in the treatment of this deadly disease and it was established without any doubt that the disease could be cured completely provided the patient took the treatment which was essentially a multi-drug regime in the correct doses for a definite period of time.

However, the organism which causes TB in humans, Mycobacterium Tuberculosis (MTB) is notorious for its capability to evade elimination. Mutations develop in MTB which can make the mutant strain inherently resistance to an anti-tubercular drug. Fortunately such natural mutations are rare and the combination of drugs ensures that if one is ineffective, the others will act and destroy the mutant strain. But factors such as a poorly designed regime, wrong dosages, non-compliance and incomplete duration, either acted alone or in unison to trigger the selection of the resistant mutants and Multi-drug Resistant (MDR) TB developed (Resistance to at least Isoniazid and Rifampicin).

MDR TB with resistance to Second line drugs (SLI) or Fluoroquinolones (FQ) or both i.e. Extensive Drug Resistant (XDR) TB are now rampant worldwide with India having the greatest number of MDR TB patients ( $25 \%$ of the world). The management of MDR TB with increasing levels of resistance is fraught with difficulties as the drug regime includes second line agents which are less effective, more toxic and expensive. The drug treatment of MDR TB has received some boost by the discovery of drugs such as bedaquiline (BDQ) with its novel mechanism of action involving inhibition of Mycobacterial ATP Synthase.

Recently there has been a revival of interest in the role of Thoracic Surgery in the management of MDR TB. As mentioned in the beginning, Surgery had played a significant role in the overall management of TB in the pre-antiboitic era but with the advent of highly effective chemotherapy, it was relegated to a comparatively insignificant position. 


\section{Open Access Journal of Pulmonary \& Respiratory Sciences}

However, as we will see now, Surgery has once more emerged as an important tool in the overall management of TB, especially DR-TB. In-spite of its increasing importance in the armamentarium against DR-TB, the role of Surgery is adjuvant and full support of an adequate anti-tubercular drug regime is paramount.

These may be considered the eligible cases in which Surgery may play a rather important part in the holistic management of DR-TB though the list is not exhaustive [2].

1. Patients with adequate pulmonary reserve and localized disease.

2. Patients whom in spite of an adequate trial of appropriate chemotherapy remain persistently positive as far as sputum smears and/or cultures are concerned.

3. Patients in whom the risk of relapse is very high (this being inferred by the results of Drug Resistance Tests and/or radiographic details).

4. Bronchial Stump Healing is less relevant as drug resistance profile is rather high and thus Surgery is the only option

5. Decortication in case of Empyema in which DR-TB has been diagnosed based on molecular tests or phenotypic method.

A very crucial factor that has to be considered in such cases is the timing for the Surgery. Ideally Surgery should be performed when the patient is culture negative and his general condition including nutritional status is fair. But in patients with DR-TB especially in the more advanced forms, this ideal condition may never be achieved and delaying surgery in the false hopes of a drug cure or even remission may further unleash the destructive consumption of this terrible affliction leading to progressive parenchymal scarring and damage to the lungs [3]. However, it is desirable that at least 3 to 6 months of chemotherapy should be administered to the patient before embarking on to Surgery [4].

The surgery employed should remove as much damaged lung as possible while preserving as much healthy tissue as feasible. Pneumonectomies, lobectomies, segmental resection and cavity removal bilaterally in sequence are all described [5]. New techniques such as Video Assisted Thoracic Surgery (VATS) have recently, in carefully selected patients with less extensive disease, been shown to be a feasible option for isolated lobectomy and smaller wedge resection. Moreover, these have been associated with less wound pain, fewer pulmonary complications and a shorter hospital stay than with a thoracotomy [6].

Though it is difficult to exactly predict the proportion of Success in the management of DR-TB cases which can be attributed to Surgery, yet some literature does provide verity to the assumption that Surgical intervention in addition to Medical management does improve the chances of a favourable outcome in DR-TB patients [7]. This underscores the importance of the Surgeon's role in the management of DR-TB, with increasing levels and complexity of the resistance pattern.

In conclusion, in the present era of management of cases of DR-TB, with increasing levels and complexity of resistance, the role of Surgery as an adjuvant can improve the overall outcome of management which of course should be based on the most suitable and well-designed drug regime. Hence the Surgeon should also be an integral part of the multispecialty team dealing with the holistic management of Drug Resistant Tuberculosis.

\section{References}

1. Perelman MI, Strelzov VP (1997) Surgery for pulmonary tuberculosis. World J Surg 21(5): 457-467.

2. Iseman MD, Madsen L, Goble M, Pomerantz M (1990) Surgical intervention in the treatment of pulmonary disease caused by drug-resistant Mycobacterium Tuberculosis. Am Rev Respir Dis 141: 623-625.

3. Man MA, Nicolau D (2012) Surgical treatment to increase the success rate of multi drug-resistant tuberculosis. Eur J Cardiothorac Surg 42(1): e9-12.

4. Pomerantz BJ, Cleveland JC, Olson HK, Pomerantz M (2001) Pulmonary resection for multi-drug resistant tuberculosis. J Thorac Cardiovasc Surg 121(3): 448453.

5. Gegia M, Kalandadze I, Kempker RR, Magee MJ, Blumberg HM, etal. (2012) Adjunctive surgery improves treatment outcomes among patients with multidrugresistant and extensively drug-resistant tuberculosis. Int J Infect Dis 16(5): E391-E396.

6. Yen YT, Wu MH, Lai WW, Chang JM, Hsu IL, et al. (2013) The role of video-assisted thoracoscopic surgery in therapeutic lung resection for pulmonary tuberculosis. Ann Thorac Surg 95(1): 257-263.

7. Marrone MT, Venkataramanan V, Goodman M, Hill AC, Jereb JA, et al. (2013) Surgical interventionsfor drugresistant tuberculosis: a systematic review and metaanalysis. Int J Tuberc Lung Dis 17(1): 6-16. 